\title{
Smoothed Analysis of Binary Search Trees and Quicksort Under Additive Noise
}

\author{
Bodo Manthey ${ }^{1}$, Till Tantau ${ }^{2}$ \\ 1 Universität des Saarlandes, Informatik \\ Postfach 151150, 66041 Saarbrücken, Germany \\ manthey@cs.uni-sb.de \\ 2 Universität zu Lübeck, Institut für Theoretische Informatik \\ Ratzeburger Allee 160, 23538 Lübeck, Germany \\ tantau@tcs.uni-luebeck.de
}

\begin{abstract}
Binary search trees are a fundamental data structure and their height plays a key role in the analysis of divide-and-conquer algorithms like quicksort. Their worst-case height is linear; their average height, whose exact value is one of the best-studied problems in averagecase complexity, is logarithmic. We analyze their smoothed height under additive noise: An adversary chooses a sequence of $n$ real numbers in the range $[0,1]$, each number is individually perturbed by adding a random value from an interval of size $d$, and the resulting numbers are inserted into a search tree. The expected height of this tree is called the smoothed tree height. If $d$ is very small, namely for $d \leq 1 / n$, the smoothed tree height is the same as the worst-case height; if $d$ is very large, the smoothed tree height approaches the logarithmic average-case height. An analysis of what happens between these extremes lies at the heart of our paper: We prove that the smoothed height of binary search trees is $\Theta(\sqrt{n / d}+\log n)$, where $d \geq 1 / n$ may depend on $n$. This implies that the logarithmic average-case height becomes manifest for $d \in \Omega\left(n / \log ^{2} n\right)$. For the analysis, we first prove that the smoothed number of left-to-right maxima in a sequence is also $\Theta(\sqrt{n / d}+\log n)$. We apply these findings to the performance of the quicksort algorithm and prove that the smoothed number of comparisons made by quicksort is $\Theta\left(\frac{n}{d+1} \sqrt{n / d}+n \log n\right)$.
\end{abstract}

Keywords. Smoothed analysis, binary search trees, quicksort, left-toright maxima

\section{Introduction}

To explain the discrepancy between average-case and worst-case behavior of the simplex algorithm, Spielman and Teng introduced the notion of smoothed analysis [11]. Smoothed analysis interpolates between average-case and worst-case analysis: Instead of taking a worst-case instance or, as in average-case analysis, choosing an instance completely at random, we analyze the complexity of (possibly worst-case) objects subject to slight random perturbations. On the one

Dagstuhl Seminar Proceedings 07391

Probabilistic Methods in the Design and Analysis of Algorithms

http://drops.dagstuhl.de/opus/volltexte/2007/1289 
hand, perturbations model that nature is not (or not always) adversarial. On the other hand, perturbations reflect the fact that data is often subject to measurement or rounding errors; even if the instance at hand was initially a worst-case instance, due to such errors we would probably get a less difficult instance in practice. Spielman and Teng [12] give a comprehensive survey on results and open problems in smoothed analysis.

Binary search trees are one of the most fundamental data structures in computer science and they are the building blocks for a large variety of data structures. The most important parameter of binary search trees is their height. The worst-case height of a binary tree for $n$ numbers is $n$. The average-case behavior has been the subject of a considerable amount of research, culminating in the result that the average-case height is $\alpha \ln n-\beta \ln \ln n+O(1)$, where $\alpha \approx 4.311$ is the larger root of $\alpha \ln (2 e / \alpha)=1$ and $\beta=3 /(2 \ln (\alpha / 2)) \approx 1.953$ [8]. Furthermore, the variance of the height is constant, as was proved independently by Drmota [2] and Reed [8], and it is conjectured that all moments are bounded by constants as well [9]. Drmota [3] gives a recent survey.

Beyond being an important data structure, binary search trees play a central role in the analysis of divide-and-conquer algorithms like quicksort [5, Section 5.2.2]. While quicksort needs $\Theta\left(n^{2}\right)$ comparisons in the worst case, the average number of comparisons is $2 n \log n-\Theta(n)$ with a variance of $\left(7-\frac{2}{3} \pi^{2}\right)$. $n^{2}-2 n \log n+O(n)$ as mentioned by Fill and Janson [4]. Quicksort and binary search trees are closely related: The height of the tree $T(\sigma)$ obtained from a sequence $\sigma$ is equal to the number of levels of recursion required by quicksort to sort $\sigma$. The number of comparisons, which corresponds to the total path length of $T(\sigma)$, is at most $n$ times the height of $T(\sigma)$.

Binary search trees are also related to the number of left-to-right maxima of a sequence, which is the number of new maxima seen while scanning a sequence from left to right. The number of left-to-right maxima of $\sigma$ is equal to the length of the rightmost path of the tree $T(\sigma)$, which means that left-to-right maxima provide an easy-to-analyze lower bound for the height of binary search trees. Furthermore, left-to-right maxima play a role in the analysis of quicksort [10]. In the worst-case, the number of left-to-right maxima is $n$, while it is $\sum_{i=1}^{n} 1 / i \in$ $\Theta(\log n)$ on average.

Given the discrepancies between average-case and worst-case behavior of binary search trees, quicksort, and the number of left-to-right maxima, the question arises of what happens in between when the randomness is limited.

Previously Studied Perturbation Models. The perturbation model introduced by Spielman and Teng for the smoothed analysis of continuous problems like linear programming is appropriate for algorithms that process real numbers. In their model, each of the real numbers in the adversarial input is perturbed by adding a small Gaussian noise. This model of perturbation favors instances in the neighborhood of the adversarial input for a fairly natural and realistic notion of "neighborhood." 
The first smoothed analysis of quicksort, due to Banderier, Beier, and Mehlhorn [1], uses a different perturbation model, namely a discrete perturbation model. Such models take discrete objects like permutations as input and again yield discrete objects like another permutation. Banderier et al. used p-partial permutations, which work as follows: An adversary chooses a permutation of the numbers $\{1, \ldots, n\}$ as sequence, every element of the sequence is marked independently with a probability of $p$, and then the marked elements are randomly permuted. Banderier et al. showed that the number of comparisons subject to $p$-partial permutations is $O\left(\frac{n}{p} \cdot \log n\right)$. Furthermore, they proved bounds on the smoothed number of left-to-right maxima subject to this model.

Manthey and Reischuk [6] analyzed the height of binary search trees under $p$-partial permutations. They proved a lower bound of $0.8 \cdot(1-p) \cdot \sqrt{n / p}$ and an asymptotically matching upper bound of $6.7 \cdot(1-p) \cdot \sqrt{n / p}$ for smoothed tree height. For the number of left-to-right maxima they showed a lower bound of $0.6 \cdot(1-p) \cdot \sqrt{n / p}$ and an upper bound of $3.6 \cdot(1-p) \cdot \sqrt{n / p}$.

Special care must be taken when defining perturbation models for discrete inputs: The perturbation should favor instances in the neighborhood of the adversarial instance, which requires a suitable definition of neighborhood in the first place, and the perturbation should preserve the global structure of the adversarial instance. Partial permutations have the first feature [6, Lemma 3.2], but destroy much of the global order of the adversarial sequence.

Our Perturbation Model and Our Results. We continue the smoothed analysis of binary search trees and quicksort begun by Banderier et al. [1] and Manthey and Reischuk [6]. However, we return to the original idea of smoothed analysis that input numbers are perturbed by adding random numbers. In our model the adversarial input sequence consists of $n$ real numbers in the interval $[0,1]$. Then, each of the real numbers is individually perturbed by adding a random number drawn uniformly from an interval of size $d$. If $d<1 / n$, then the sorted sequence $(1 / n, 2 / n, 3 / n, \ldots, n / n)$ stays a sorted sequence. This means that for $d<1 / n$ the smoothed height of binary search trees (as well as the performance of quicksort and the number of left-to-right maxima) is the same as in the worst-case. For this reason, we always assume $d \geq 1 / n$ in the following.

We study the smoothed height of binary search trees, the smoothed number of comparisons made by quicksort, and the smoothed number of left-to-right maxima under additive noise. In each case we prove tight upper and lower bounds:

1. The smoothed number of left-to-right maxima is $\Theta(\sqrt{n / d}+\log n)$ as shown in Section 3. This result will be exploited in the subsequent sections.

2. The smoothed height of binary search trees is $\Theta(\sqrt{n / d}+\log n)$ as shown in Section 4.

3. The smoothed number of comparisons made by quicksort is $\Theta\left(\frac{n}{d+1} \sqrt{n / d}+\right.$ $n \log n)$ as shown in Section 5. Thus, the perturbation effect of $d \in \omega(1)$ is stronger than for $d \in o(1)$.

Already for $d \in \omega(1 / n)$, we obtain bounds that are asymptotically better than the worst-case bounds. For constant values of $d$, which correspond to a perturba- 
tion by a constant percentage like 10\%, the height of binary search trees drops from the worst-case height of $n$ to $O(\sqrt{n})$, and quicksort needs only $O\left(n^{3 / 2}\right)$ comparisons.

\section{Preliminaries}

Intervals of the real axis are denoted by $[a, b]=\{x \in \mathbb{R} \mid a \leq x \leq b\}$. To denote an interval that does not include an endpoint, we replace the square bracket next to the endpoint by a parenthesis. We denote sequences of real numbers by $\sigma=\left(\sigma_{1}, \ldots, \sigma_{n}\right)$, where $\sigma_{i} \in \mathbb{R}$. For $U=\left\{i_{1}, \ldots, i_{\ell}\right\} \subseteq\{1, \ldots, n\}$ with $i_{1}<i_{2}<\cdots<i_{\ell}$ let $\sigma_{U}=\left(\sigma_{i_{1}}, \sigma_{i_{2}}, \ldots, \sigma_{i_{\ell}}\right)$ denote the subsequence of $\sigma$ of the elements at positions in $U$.

We denote probabilities by $\mathbb{P}$ and expected values by $\mathbb{E}$. To bound large deviations from the expected value, we will use the Chernoff bound [7, Sect. 4.1] a couple of times: Let $X_{1}, \ldots, X_{n}$ be random variables with $\mathbb{P}\left(X_{i}=1\right)=p=$ $1-\mathbb{P}\left(X_{i}=0\right)$. Let $X=\sum_{i=1}^{n} X_{i}$. Then $\mathbb{E}(X)=p n$ and, for every $\delta>0$, we have

$$
\mathbb{P}(X>(1+\delta) \cdot p n)<\left(\frac{\exp (\delta)}{(1+\delta)^{1+\delta}}\right)^{p n} .
$$

Throughout the paper, we will assume for the sake of clarity that numbers like $\sqrt{d}$ are integers and we do not write down the tedious floor and ceiling functions that are actually necessary. Since we are interested in asymptotic bounds, this does not affect the validity of the proofs.

\subsection{Binary Search Trees, Left-To-Right Maxima, and Quicksort}

Let $\sigma$ be a sequence of length $n$ consisting of pairwise distinct elements. For the following definitions, let $G=\left\{i \in\{1, \ldots, n\} \mid \sigma_{i}>\sigma_{1}\right\}$ be the set of positions of elements greater than $\sigma_{1}$, and let $S=\left\{i \in\{1, \ldots, n\} \mid \sigma_{i}<\sigma_{1}\right\}$ be the set of positions of elements smaller than $\sigma_{1}$.

From $\sigma$, we obtain a binary search tree $T(\sigma)$ by iteratively inserting the elements $\sigma_{1}, \sigma_{2}, \ldots, \sigma_{n}$ into the initially empty tree as follows: The root of $T(\sigma)$ is $\sigma_{1}$. The left subtree of the root $\sigma_{1}$ is $T\left(\sigma_{S}\right)$, and the right subtree of $\sigma_{1}$ is $T\left(\sigma_{G}\right)$. The height of $T(\sigma)$ is the maximum number of nodes on any root-toleaf path of $T(\sigma)$ : Let height $(\sigma)=1+\max \left\{\operatorname{height}\left(\sigma_{S}\right)\right.$, height $\left.\left(\sigma_{G}\right)\right\}$, and let height $(\sigma)=0$ when $\sigma$ is the empty sequence.

The number of left-to-right maxima of $\sigma$ is the number of maxima seen when scanning $\sigma$ from left to right: let $\operatorname{ltrm}(\sigma)=1+\operatorname{ltrm}\left(\sigma_{G}\right)$, and $\operatorname{let} \operatorname{ltrm}(\sigma)=0$ when $\sigma$ is the empty sequence. The number of left-to-right maxima of $\sigma$ is equal to the length of the rightmost path of $T(\sigma)$, so $\operatorname{ltrm}(\sigma) \leq \operatorname{height}(\sigma)$.

Quicksort is the following sorting algorithm: Given $\sigma$, we construct $\sigma_{S}$ and $\sigma_{G}$. To do this, all elements of $\left(\sigma_{2}, \ldots, \sigma_{n}\right)$ have to be compared to $\sigma_{1}$, which is called the pivot element. Then we sort $\sigma_{S}$ and $\sigma_{G}$ recursively to obtain $\tau_{S}$ and $\tau_{G}$, respectively. Finally, we output $\tau=\left(\tau_{S}, \sigma_{1}, \tau_{G}\right)$. The number qs $(\sigma)$ of comparisons needed to sort $\sigma$ is thus $\mathrm{qs}(\sigma)=(n-1)+\mathrm{qs}\left(\sigma_{S}\right)+\mathrm{qs}\left(\sigma_{G}\right)$ if $\sigma$ has a length of $n \geq 1$, and $\mathrm{qs}(\sigma)=0$ when $\sigma$ is the empty sequence. 


\section{$2.2 \quad$ Perturbation Model}

The perturbation model of additive noise is defined as follows: Let $d=d(n) \geq 0$ be the perturbation parameter ( $d$ may depend on $n$ ). Given a sequence $\sigma$ of $n$ numbers chosen by an adversary from the interval $[0,1]$, we draw a noise $\nu_{i}$ for each $i \in\{1, \ldots, n\}$ uniformly at random from the interval $[0, d]$. Then we obtain the perturbed sequence $\bar{\sigma}=\left(\bar{\sigma}_{1}, \ldots, \bar{\sigma}_{n}\right)$ by adding $\nu_{i}$ to $\sigma_{i}$, that is, $\bar{\sigma}_{i}=\sigma_{i}+\nu_{i}$. Note that $\bar{\sigma}_{i}$ need no longer be an element of $[0,1]$, but $\bar{\sigma}_{i} \in[0, d+1]$. For $d>0$ all elements of $\bar{\sigma}$ are distinct with a probability of 1 .

For this perturbation model, we define the random variables height ${ }_{d}(\sigma)$, $\mathrm{qs}_{d}(\sigma)$, and $\operatorname{ltrm}_{d}(\sigma)$, which denote the smoothed search tree height, smoothed number of quicksort comparisons, and smoothed number of left-to-right maxima, respectively, when $\sigma$ is perturbed by $d$-noise. Since the adversary chooses $\sigma$, our goal is to bound $\max _{\sigma \in[0,1]^{n}} \mathbb{E}\left(\right.$ height $\left._{d}(\sigma)\right)$, $\max _{\sigma \in[0,1]^{n}} \mathbb{E}\left(\mathrm{qs}_{d}(\sigma)\right)$, and $\max _{\sigma \in[0,1]^{n}} \mathbb{E}\left(\operatorname{ltrm}_{d}(\sigma)\right)$. In the following, we will sometimes write height $(\bar{\sigma})$ instead of height $d(\sigma)$ if $d$ is clear from the context. Since $\bar{\sigma}$ is random, height $(\bar{\sigma})$ is also a random variable. Similarly, we will use $\operatorname{ltrm}(\bar{\sigma})$ and $\mathrm{qs}(\bar{\sigma})$.

The choice of the interval sizes is arbitrary since the model is invariant under scaling if we scale the perturbation parameter accordingly. This is summarized in the following lemma, which we will exploit a couple of times in the following.

Lemma 1. Let $b>a$ and $d>0$ be arbitrary real numbers, and let $d^{\prime}=d /(b-a)$. Then

$$
\max _{\sigma \in[a, b]^{n}} \mathbb{E}\left(\text { height }_{d}(\sigma)\right)=\max _{\sigma \in[0,1]^{n}} \mathbb{E}\left(\text { height }_{d^{\prime}}(\sigma)\right) .
$$

For quicksort and the number of left-to-right maxima, we have analogous equalities.

As argued earlier, if $d<1 / n$, the adversary can specify the sequence $\sigma=$ $(1 / n, 2 / n, 3 / n, \ldots, n / n)$ and adding the noise terms does not affect the order of the elements. This means that we get the worst-case height, number of comparisons, and number of left-to-right maxima. Because of this observation we will restrict our attention to $d \geq 1 / n$.

If $d$ is large, the noise will swamp out the original instance, and the order of the elements of $\bar{\sigma}$ will depend only on the noise rather than the original instance. For intermediate $d$, additive noise interpolates between average and worst case.

\section{Smoothed Number of Left-To-Right Maxima}

We start our analyses with the smoothed number of left-to-right maxima, which provides us with a lower bound on the height of binary search trees as well. Our aim for the present section is to prove the following theorem.

Theorem 1. For $d \geq 1 / n$, we have

$$
\max _{\sigma \in[0,1]^{n}} \mathbb{E}\left(\operatorname{ltrm}_{d}(\sigma)\right) \in \Theta(\sqrt{n / d}+\log n) .
$$


The theorem is proved in the rest of this section by first proving an upper bound and then proving a lower bound. In the proofs, the following notations will be helpful: For $j \leq 0$, let $\sigma_{j}=\nu_{j}=0$. This allows us to define $\delta_{i}=\sigma_{i}-\sigma_{i-\sqrt{n d}}$ for all $i \in\{1, \ldots, n\}$. We define $I_{i}=\{j \in\{1, \ldots, n\} \mid i-\sqrt{n d} \leq j<i\}$ to be the set of the $\left|I_{i}\right|=\min \{i-1, \sqrt{n d}\}$ positions that precede $i$.

\subsection{Upper Bound on the Smoothed Number of Left-To-Right Maxima}

To prove the upper bound for the smoothed number of left-to-right maxima, we proceed in two steps: First, a "bubble-sorting argument" is used to show that the adversary should choose a sorted sequence. Second, we prove that the expected number of left-to-right maxima of sorted sequences is $O(\sqrt{n / d}+\log n)$.

Lemma 2. For every sequence $\sigma$ and its sorted version $\tau$, we have

$$
\mathbb{E}\left(\operatorname{ltrm}_{d}(\sigma)\right) \leq \mathbb{E}\left(\operatorname{ltrm}_{d}(\tau)\right)
$$

Proof. We prove the lemma by "bubble-sorting" $\sigma$. If $\sigma$ is already sorted, then there is nothing to show. Otherwise, there exist adjacent $\sigma_{i}$ and $\sigma_{i+1}$ with $\sigma_{i}>$ $\sigma_{i+1}$. Our aim is to show that $\mathbb{E}\left(\operatorname{ltrm}_{d}(\sigma)\right) \leq \mathbb{E}\left(\operatorname{ltrm}_{d}(\tau)\right)$, where $\tau$ is obtained from $\sigma$ by swapping $\sigma_{i}$ and $\sigma_{i+1}$. Then the claim follows by iteratively applying this argument.

After perturbation with $\nu$, we obtain $\bar{\sigma}$ and $\bar{\tau}$, where $\bar{\tau}_{i}=\tau_{i}+\nu_{i+1}=$ $\sigma_{i+1}+\nu_{i+1}$ and $\bar{\tau}_{i+1}=\tau_{i+1}+\nu_{i}=\sigma_{i}+\nu_{i}$. Now we analyze the number of leftto-right maxima of $\bar{\sigma}$ and $\bar{\tau}$. To do this, let $\delta=\sigma_{i}-\sigma_{i+1}>0$. We distinguish two cases. First, we condition on $\nu_{i} \in[0, d-\delta]$ and $\nu_{i+1} \in[\delta, d]$. In this case, both $\left(\bar{\sigma}_{i}, \bar{\sigma}_{i+1}\right)$ and $\left(\bar{\tau}_{i}, \bar{\tau}_{i+1}\right)$ are pairs of random numbers, all of which lie uniformly in the interval $\left[\sigma_{i}, \sigma_{i+1}+d\right]$. Then the expected numbers of left-to-right maxima of $\bar{\sigma}$ and $\bar{\tau}$ are equal. Second, we condition on the event that $\nu_{i} \in(d-\delta, d]$ or $\nu_{i+1} \in[0, \delta)$. In either case, both $\bar{\sigma}_{i}>\bar{\sigma}_{i+1}$ and $\bar{\tau}_{i}<\bar{\tau}_{i+1}$ hold. Then $\bar{\sigma}_{i+1}$ cannot be a left-to-right maximum in $\bar{\sigma}$, and if $\bar{\sigma}_{i}$ is a left-to-right maximum in $\bar{\sigma}$, then so is $\bar{\tau}_{i+1}$ in $\bar{\tau}$. Since the case distinction is exhaustive, the lemma is proved.

Lemma 3. For every sequence $\sigma$ of length $n$ and all $d \geq 1 / n$, we have

$$
\mathbb{E}\left(\operatorname{ltrm}_{d}(\sigma)\right) \in O(\sqrt{n / d}+\log n)
$$

Proof. By Lemma 2 we can restrict ourselves to proving the lemma for sorted sequences $\sigma$. We estimate the probability that a given $\bar{\sigma}_{i}$ for $i \in\{1, \ldots, n\}$ is a left-to-right maximum. Then the bound follows by the linearity of expectation. To bound the probability that $\bar{\sigma}_{i}$ is a left-to-right maximum (ltrm), consider the 
following computation:

$$
\begin{aligned}
& \mathbb{P}\left(\bar{\sigma}_{i} \text { is an ltrm }\right) \\
\leq & \mathbb{P}\left(\forall j \in I_{i}: \nu_{j}<\bar{\sigma}_{i}-\sigma_{i-\sqrt{n d}}\right) \\
\leq & \mathbb{P}\left(d<\bar{\sigma}_{i}-\sigma_{i-\sqrt{n d}}\right)+\int_{0}^{d-\delta_{i}} \mathbb{P}\left(\forall j \in I_{i}: \nu_{j}<\sigma_{i}+x-\sigma_{i-\sqrt{n d}}\right) \mathrm{d} x \\
\leq & \frac{\delta_{i}}{d}+\int_{0}^{d} \mathbb{P}\left(\forall j \in I_{i}: \nu_{j}<x\right) \mathrm{d} x \\
\leq & \frac{\delta_{i}}{d}+\mathbb{P}\left(\forall j \in I_{i}: \nu_{j}<\nu_{i}\right)=\frac{\delta_{i}}{d}+\frac{1}{\left|I_{i}\right|+1} .
\end{aligned}
$$

To see that (1) holds, assume that $\bar{\sigma}_{i}$ is a left-to-right maximum. Then $\bar{\sigma}_{i}-$ $\sigma_{i-\sqrt{n d}}$ must be larger than the noises of all the elements in the index range $I_{i}$, for if the noise $\nu_{j}$ of some element $\sigma_{j}$ were larger than $\bar{\sigma}_{i}-\sigma_{i-\sqrt{n d}}$, then $\bar{\sigma}_{j}=\sigma_{j}+\nu_{j}$ would be larger than $\sigma_{j}+\bar{\sigma}_{i}-\sigma_{i-\sqrt{n d}}$. Since the sequence is sorted, we would get $\sigma_{j}+\bar{\sigma}_{i}-\sigma_{i-\sqrt{n d}} \geq \bar{\sigma}_{i}$, and $\bar{\sigma}_{i}$ would not be a left-to-right maximum.

For (2), first observe that $\nu_{j}<\bar{\sigma}_{i}-\sigma_{i-\sqrt{n d}}$ is surely the case for all $j \in I_{i}$ if $d<\bar{\sigma}_{i}-\sigma_{i-\sqrt{n d}}$. So, consider the case $d \geq \bar{\sigma}_{i}-\sigma_{i-\sqrt{n d}}=\delta_{i}+\nu_{i}$. Then

$\nu_{i} \in\left[0, d-\delta_{i}\right]$ and we can rewrite $\mathbb{P}\left(\forall j \in I_{i}: \nu_{j}<\delta_{i}+\nu_{i}\right)$ as $\int_{0}^{d-\delta_{i}} \mathbb{P}(\forall j \in$ $\left.I_{i}: \nu_{j}<\delta_{i}+x\right) \mathrm{d} x$. For (3) observe that $d<\bar{\sigma}_{i}-\sigma_{i-\sqrt{n d}}$ is equivalent to $d-\delta_{i}<\nu_{i}$ and the probability of this is $\delta_{i} / d$. Furthermore, we performed an index shift in the integral. In (4), we replaced the integral by a probability once more and get the final result.

We have $\sum_{i=1}^{n} \delta_{i}=\sum_{i=1}^{n}\left(\sigma_{i}-\sigma_{i-\sqrt{n d}}\right)=\sum_{i=n-\sqrt{n d}+1}^{n} \sigma_{i} \leq \sqrt{n d}$. The second equality holds since most $\sigma_{i}$ cancel themselves out and $\sigma_{i}=0$ for $i \leq 0$. The inequality holds since there are $\sqrt{n d}$ summands. We complete the proof by bounding $1 /\left(\left|I_{i}\right|+1\right)=1 / \min \{i, \sqrt{n d}+1\}$ by $1 / i+1 / \sqrt{n d}$ and summing over all $i$ :

$$
\mathbb{E}\left(\operatorname{ltrm}_{d}(\sigma)\right) \leq \sum_{i=1}^{n} \frac{\delta_{i}}{d}+\sum_{i=1}^{n} \frac{1}{\left|I_{i}\right|+1} \leq \frac{\sqrt{n d}}{d}+\sum_{i=1}^{n} \frac{1}{i}+\frac{n}{\sqrt{n d}} \in O(\sqrt{n / d}+\log n) .
$$

\subsection{Lower Bound on the Smoothed Number of Left-To-Right Maxima}

Let us now show a lower bound that matches the upper bound proved in the previous section. Although the sequences may consist of $n$ arbitrary numbers from the interval $[0,1]$, it suffices to consider the sorted sequence $(1 / n, 2 / n, \ldots, n / n)$.

Lemma 4. For the sequence $\sigma=(1 / n, 2 / n, \ldots, n / n)$ and all $d \geq 1 / n$, we have

$$
\mathbb{E}\left(\operatorname{ltrm}_{d}(\sigma)\right) \in \Omega(\sqrt{n / d}+\log n) .
$$


Proof. We assume that $d \geq 4 / n$. This is no restriction since for $d<4 / n$, we immediately get a lower bound of $n / 4 \in \Omega(n)$ in compliance with the theorem.

We give two estimates for the probability that a given $\bar{\sigma}_{i}$ is a left-to-right maximum; only this time, we need to bound this probability from below. The first estimate is simple:

$\mathbb{P}\left(\bar{\sigma}_{i}\right.$ is an ltrm $)=\mathbb{P}\left(\forall j<i: \nu_{j}+j / n<\nu_{i}+i / n\right) \geq \mathbb{P}\left(\forall j<i: \nu_{j}<\nu_{i}\right)=1 / i$.

For the second estimate, assume $\nu_{i}>d-\sqrt{d / n}$ for a given $i \in\{1, \ldots, n\}$. Then $\bar{\sigma}_{j}<\bar{\sigma}_{i}$ for all $j \in\{1, \ldots, i-\sqrt{n d}-1\}$ since the noise of $\sigma_{i}$ is so large that $\sigma_{j}$ before $\sigma_{i-\sqrt{n d}}$ can never reach $\bar{\sigma}_{i}$ even when a noise of $d$ is added. This shows that $\bar{\sigma}_{i}$ must be a left-to-right maximum if (a) we have $\nu_{i}>d-\sqrt{d / n}$ and (b) we have $\nu_{j}<d-\sqrt{d / n}$ for all $j \in I_{i}$. The probability of (a) is $1 / \sqrt{n d}$ and of (b) is $(1-1 / \sqrt{n d})^{\left|I_{i}\right|} \geq(1-1 / \sqrt{n d})^{\sqrt{n d}}$. Since $d \geq 4 / n$, this yields

$$
\mathbb{P}\left(\bar{\sigma}_{i} \text { is an ltrm }\right) \geq \frac{1}{\sqrt{n d}}\left(1-\frac{1}{\sqrt{n d}}\right)^{\sqrt{n d}} \geq \frac{1}{4 \cdot \sqrt{n d}} .
$$

The two estimates together yield $\mathbb{P}\left(\bar{\sigma}_{i}\right.$ is an ltrm $) \geq \max \{1 / i, 1 /(4 \sqrt{n d})\} \geq$ $\frac{1}{2}(1 / i+1 /(4 \sqrt{n d}))$. By the linearity of expectation we get

$$
\mathbb{E}\left(\operatorname{ltrm}_{d}(\sigma)\right) \geq \frac{1}{2} \sum_{i=1}^{n} \frac{1}{i}+\frac{1}{2} \sum_{i=1}^{n} \frac{1}{4 \sqrt{n d}} \in \Theta(\log n+\sqrt{n / d}) .
$$

\section{Smoothed Height of Binary Search Trees}

In this section we prove our first main result, an exact bound on the smoothed height of binary search trees under additive noise. The bound is the same as for left-to-right maxima, as stated in the following theorem.

Theorem 2. For $d \geq 1 / n$, we have

$$
\max _{\sigma \in[0,1]^{n}} \mathbb{E}\left(\operatorname{height}_{d}(\sigma)\right) \in \Theta(\sqrt{n / d}+\log n) .
$$

In the rest of this section, we prove this theorem. We have to prove an upper and a lower bound, but the lower bound follows directly from the lower bound of $\Omega(\sqrt{n / d}+\log n)$ for the smoothed number of left-to-right maxima (the number of left-to-right maxima in a sequence is the length of the rightmost path of the sequence's search tree). Thus, we only need to focus on the upper bound. To prove the upper bound of $O(\sqrt{n / d}+\log n)$ on the smoothed height of binary search trees, we need some preparations. In the next subsection we introduce the concept of increasing and decreasing runs and show how they are related to binary search tree height. As we will see, bounding the length of these runs implicitly bounds the height of binary search trees. This allows us to prove the upper bound on the smoothed height of binary search trees in the main part of this section. 


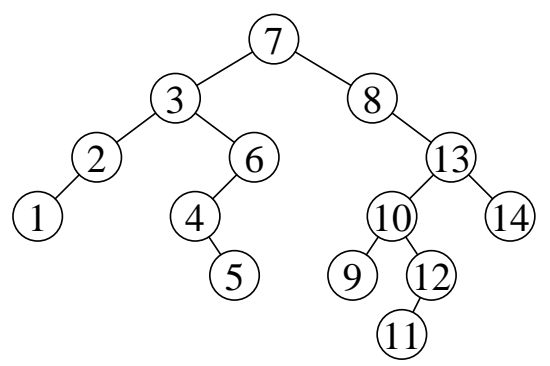

Fig. 1. The tree $T(\sigma)$ obtained from $\sigma=(7,8,13,3,2,10,9,6,4,12,14,1,5,11)$. We have height $(\sigma)=6$. The root-to-leaf path ending at 11 can be divided into the increasing run $\alpha=(7,8,10,11)$ and the decreasing run $\beta=(13,12,11)$.

\subsection{Increasing and Decreasing Runs}

In order to analyze the smoothed height of binary search trees, we introduce a related measure for which an upper bound is easier to obtain. Given a sequence $\sigma$, consider a root-to-leaf path of the tree $T(\sigma)$. We extract two subsequences $\alpha=$ $\left(\alpha_{1}, \ldots, \alpha_{k}\right)$ and $\beta=\left(\beta_{1}, \ldots, \beta_{\ell}\right)$ from this path according to the following algorithm: We start at the root. When we are at an element $\sigma_{i}$ of the path, we look at the direction in which the path continues from $\sigma_{i}$. If it continues with the right child of $\sigma_{i}$, we append $\sigma_{i}$ to $\alpha$; if it continues with the left child, we append $\sigma_{i}$ to $\beta$; and if $\sigma_{i}$ is a leaf (has no children), then we append $\sigma_{i}$ to both $\alpha$ and $\beta$. This construction ensures $\alpha_{1}<\cdots<\alpha_{k}=\beta_{\ell}<\cdots<\beta_{1}$ and the length of $\sigma$ is $k+\ell-1$. Figure 1 shows an example of how $\alpha$ and $\beta$ are constructed.

A crucial property of the sequence $\alpha$ is the following: Let $\alpha_{i}=\sigma_{j_{i}}$ for all $i \in\{1, \ldots, k\}$ with $j_{1}<j_{2}<\cdots<j_{k}$. Then none of $\sigma_{1}, \ldots, \sigma_{j_{i}-1}$ lies in the interval $\left(\alpha_{i}, \alpha_{i+1}\right)$, for otherwise $\alpha_{i}$ and $\alpha_{i+1}$ cannot be on the same root-to-leaf path. A similar property holds for the sequence $\beta$ : No element of $\sigma$ prior to $\beta_{i}$ lies in the interval $\left(\beta_{i+1}, \beta_{i}\right)$. We introduce a special name for sequences with this property.

Definition 1. An increasing run of $\sigma$ is a subsequence $\left(\sigma_{i_{1}}, \sigma_{i_{2}}, \ldots, \sigma_{i_{k}}\right)$ with the following property: For every $j \in\{1, \ldots, k-1\}$, no element of $\sigma$ prior to $\sigma_{i_{j}}$ lies in the interval $\left(\sigma_{i_{j}}, \sigma_{i_{j+1}}\right)$. Analogously, a decreasing run of $\sigma$ is a subsequence $\left(\sigma_{i_{1}}, \ldots, \sigma_{i_{\ell}}\right)$ with $\sigma_{i_{1}}>\cdots>\sigma_{i_{\ell}}$ such no element prior to $\sigma_{i_{j}}$ lies in the interval $\left(\sigma_{i_{j+1}}, \sigma_{i_{j}}\right)$.

Let $\operatorname{inc}(\sigma)$ and $\operatorname{dec}(\sigma)$ denote the length of the longest increasing and decreasing run of $\sigma$, respectively. Furthermore, let $\operatorname{dec}_{d}(\sigma)$ and $\operatorname{inc}_{d}(\sigma)$ denote the length of the longest runs under $d$-noise. In Figure 1, we have inc $(\sigma)=4$ because of $(7,8,10,12)$ or $(7,8,13,14)$ and $\operatorname{dec}(\sigma)=4$ because of $(7,3,2,1)$.

Since every root-to-leaf path can be divided into an increasing and a decreasing run, we immediately obtain the following lemma. 
Lemma 5. For every sequence $\sigma$ and all $d$ we have

$$
\begin{gathered}
\operatorname{height}(\sigma) \leq \operatorname{dec}(\sigma)+\operatorname{inc}(\sigma), \\
\mathbb{E}\left(\operatorname{height}_{d}(\sigma)\right) \leq \mathbb{E}\left(\operatorname{dec}_{d}(\sigma)+\operatorname{inc}_{d}(\sigma)\right) .
\end{gathered}
$$

In terms of upper bounds, $\operatorname{dec}(\sigma)$ and $\operatorname{inc}(\sigma)$ as well as $\operatorname{dec}_{d}(\sigma)$ and $\operatorname{inc}_{d}(\sigma)$ behave equally. The reason is that given a sequence $\sigma$, the sequence $\tau$ with $\tau_{i}=1-\sigma_{i}$ has the properties $\operatorname{dec}(\sigma)=\operatorname{inc}(\tau)$ and $\mathbb{E}\left(\operatorname{dec}_{d}(\sigma)\right)=\mathbb{E}\left(\operatorname{inc}_{d}(\tau)\right)$. This observation together with Lemma 5 proves the next lemma.

Lemma 6. For all $d$, we have

$$
\max _{\sigma \in[0,1]^{n}} \mathbb{E}\left(\text { height }_{d}(\sigma)\right) \leq 2 \cdot \max _{\sigma \in[0,1]^{n}} \mathbb{E}\left(\operatorname{inc}_{d}(\sigma)\right) .
$$

The lemma states that in order to bound the smoothed height of search trees from above we can instead bound the smoothed length of increasing or decreasing runs. To simplify the analysis even further, we show that we can once more restrict our attention to sorted sequences.

Lemma 7. For every sequence $\sigma$ and its sorted version $\tau$, we have

$$
\mathbb{E}\left(\operatorname{inc}_{d}(\sigma)\right) \leq \mathbb{E}\left(\operatorname{inc}_{d}(\tau)\right) .
$$

Proof. We sort $\sigma$ successively as we already did to prove Lemma 2. Assume that $\sigma_{i}>\sigma_{i+1}$ for some $i$ and let $\delta=\sigma_{i}-\sigma_{i+1}>0$. We show $\mathbb{E}\left(\operatorname{inc}_{d}(\sigma)\right) \leq \mathbb{E}\left(\operatorname{inc}_{d}(\tau)\right)$, where $\tau$ is obtained from $\sigma$ by swapping $\sigma_{i}$ and $\sigma_{i+1}$. Let $\nu$ denote the noise vector added to $\sigma$ and $\tau$. Then $\bar{\tau}_{i}=\tau_{i}+\nu_{i+1}=\sigma_{i+1}+\nu_{i+1}$ and $\bar{\tau}_{i+1}=\tau_{i+1}+\nu_{i}=\sigma_{i}+\nu_{i}$.

We distinguish two cases. First, we condition on $\nu_{i} \in[0, d-\delta]$ and $\nu_{i+1} \in$ $[\delta, d]$. Similar to the argument in Lemma 2 , both $\left(\bar{\sigma}_{i}, \bar{\sigma}_{i+1}\right)$ and $\left(\bar{\tau}_{i}, \bar{\tau}_{i+1}\right)$ are pairs of random numbers, all of which lie uniformly in the interval $\left[s e q_{i}, s e q_{i+1}+d\right]$, and the expected values of $\operatorname{inc}_{d}(\sigma)$ and $\operatorname{inc}_{d}(\tau)$ are equal.

Second, we condition on the events that $\nu_{i} \in(d-\delta, d]$ or $\nu_{i+1} \in[0, \delta)$. In either case, $\bar{\sigma}_{i}>\bar{\sigma}_{i+1}$ and $\bar{\tau}_{i}<\bar{\tau}_{i+1}$. Thus, every increasing run of $\bar{\sigma}$ corresponds to an increasing run of $\bar{\tau}$ : If the run of $\bar{\sigma}$ uses neither $\bar{\sigma}_{i}$ nor $\bar{\sigma}_{i+1}$, this is obvious. If the run of $\bar{\sigma}$ uses $\bar{\sigma}_{i}$, then we get the same run of $\bar{\tau}$, where now $\bar{\tau}_{i+1}$ is used. The run cannot be interrupted by $\bar{\tau}_{i}$ because $\bar{\tau}_{i}<\bar{\tau}_{i+1}$. If the run of $\bar{\sigma}$ uses $\bar{\sigma}_{i+1}$, then we obtain a run of the same length using $\bar{\tau}_{i}$. This run is also an increasing run since the only difference of $\bar{\sigma}$ and $\bar{\tau}$ is that now the larger element $\bar{\tau}_{i+1}$ appears after $\bar{\tau}_{i}$. Finally, the run of $\bar{\sigma}$ cannot use both $\bar{\sigma}_{i}$ and $\bar{\sigma}_{i+1}$ because of $\bar{\sigma}_{i+1}<\bar{\sigma}_{i}$. Thus, we have $\operatorname{inc}(\bar{\sigma}) \leq \operatorname{inc}(\bar{\tau})$, which proves the lemma.

\subsection{Upper Bound on the Smoothed Height of Binary Search Trees}

We are now ready to prove the upper bound for binary search trees by proving an upper bound on the smoothed length of increasing runs of sorted sequences. For this, we prove four lemmas, the last of which claims exactly the desired upper bound. 
Lemma 8 deals with $d=1$ and states that $\mathbb{E}\left(\right.$ height $\left._{1}(\sigma)\right) \in O(\sqrt{n})$ for every sequence $\sigma$.

Lemma 9 states that in order to bound tree heights, we can divide sequences into (possibly overlapping) parts and consider the height of the trees induced by the subsequences individually. A less general form of the lemma has already been shown by Manthey and Reischuk [6, Lemma 4.1].

Lemma 10 establishes that if $d=n / \log ^{2} n$, a perturbed sequence behaves the same way as a completely random sequence with respect to the smoothed length of its longest increasing run. The core idea is to partition the sequence into a set of "completely random" elements, which behave as expected, and two sets of more bothersome elements lying in a small range. As we will see, the number of bothersome elements is roughly $\log ^{2} n$ and since the range of values of these elements is small, we can use the result $\mathbb{E}\left(\right.$ height $\left._{1}(\sigma)\right) \in O(\sqrt{n})$ to show that their contribution to the length on an increasing run is just $O(\log n)$.

Finally, in Lemma 11 we allow general $d \geq 1 / n$. This case turns out to be reducible to the case $d=n / \log ^{2} n$ by scaling the numbers according to Lemma 1 .

For the proofs of the lemmas, two technical terms will be helpful: For a given real interval $I=[a, b]$, we say that a position $i$ of $\sigma$ is eligible for $I$ if $\bar{\sigma}_{i}$ can assume any value in $I$. In other words, $i$ is eligible for $[a, b]$ if $\sigma_{i} \leq a$ and $\sigma_{i}+d \geq b$. Furthermore, we say that $i$ is regular if $\bar{\sigma}_{i}$ actually lies inside $I$.

Lemma 8. For every sequence $\sigma$, we have

$$
\mathbb{E}\left(\operatorname{inc}_{1}(\sigma)\right) \in O(\sqrt{n}) .
$$

Proof. Let us take a closer look at increasing runs. Every increasing run of a sequence $\bar{\sigma}$ starts with a number of left-to-right maxima. However, after the first element that is not a left-to-right maximum, the run does not contain any more left-to-right maxima. More formally: If $\alpha_{1}, \ldots, \alpha_{\ell}$ is an increasing run of $\bar{\sigma}$, then there exists a $k \in\{0,1, \ldots, \ell\}$ such that $\alpha_{1}, \ldots, \alpha_{k}$ are left-to-right maxima of $\bar{\sigma}$ while $\alpha_{k+1}, \ldots, \alpha_{\ell}$ are not. Furthermore, if $\bar{\sigma}_{i}$ is the first left-to-right maximum after $\alpha_{k}$, then the remaining elements $\alpha_{k+1}, \ldots, \alpha_{\ell}$ all lie in the interval $\left[\alpha_{k}, \bar{\sigma}_{i}\right]$.

Due to this property, it suffices to a) bound $\mathbb{E}\left(\operatorname{ltrm}_{1}(\sigma)\right)$ and b) bound the maximum length of an increasing run such that the values of the elements of the run lie between the values of two consecutive left-to-right maxima. By Lemma 3, we have $\mathbb{E}\left(\operatorname{ltrm}_{1}(\sigma)\right) \in O(\sqrt{n})$, so let us focus on b). For the bound we prove two claims and for the formulation of these claims the following definition is helpful: We say that a set of numbers $x_{i}$ is $\epsilon$-dense for an interval $I$ if every interval $J \subseteq I$ of length $\epsilon$ contains at least one $x_{i}$.

Claim 1. Let $a \leq s<b$, let $y_{1}, \ldots, y_{\sqrt{n}} \in[a, s]$, and let $x_{1}, \ldots, x_{\sqrt{n}}$ be random variables where $x_{i}$ is uniformly distributed in the interval $\left[y_{i}, b\right]$. Then the $x_{i}$ are $\left((b-a) n^{-1 / 4}\right)$-dense for the interval $[s, b]$ with a probability of at least $1-\exp \left(-\Theta\left(n^{1 / 4}\right)\right)$.

Proof. By a scaling argument similar to Lemma 1, it suffices to consider the case $a=0, b=1$, and $s \in[0,1]$. We divide the interval $[s, 1]$ into subintervals 
$I_{1}, I_{2}, \ldots, I_{k}$, where

$$
I_{j}=\left[s+(j-1) \cdot n^{-1 / 4} / 2, s+j \cdot n^{-1 / 4} / 2\right]
$$

and $k=\left\lfloor(1-s) \cdot 2 n^{1 / 4}\right\rfloor$. The interval $\left[s+k \cdot n^{-1 / 4} / 2,1\right]$ is not covered by an $I_{j}$ if $(1-s) \cdot 2 n^{1 / 4}$ is not integral. But this does not cause any harm since the length of $\left[s+k \cdot n^{-1 / 4} / 2,1\right]$ is less than $n^{-1 / 4}$.

Every interval $J \subseteq[s, 1]$ of length $n^{-1 / 4}$, which is twice the length of an $I_{j}$, contains at least one $I_{j}$ as a subinterval. Thus, if every $I_{j}$ contains at least one $x_{i}$, every interval of length at least $n^{-1 / 4}$ also contains at least one $x_{i}$. The probability that any fixed element $x_{i}$ does not assume a value in $I_{j}$ is at most $1-n^{-1 / 4} / 2$. Thus, and since $1+x \leq \exp (x)$ for all $x \in \mathbb{R}$, the probability that $I_{j}$ does not contain any $x_{i}$ is at most

$$
\left(1-\frac{n^{-1 / 4}}{2}\right)^{\sqrt{n}} \leq \exp \left(-n^{1 / 4} / 2\right)
$$

Using the union bound, we obtain that the probability that there exists an empty $I_{j}$ is at most $k \cdot \exp \left(-n^{1 / 4} / 2\right) \in \exp \left(-\Theta\left(n^{1 / 4}\right)\right)$.

Claim 2. Let $\tau=\left(\tau_{1}, \ldots, \tau_{k}\right)$ be the sequence obtained by sorting $\left(\bar{\sigma}_{1}, \ldots, \bar{\sigma}_{k}\right)$, and let $\tau_{0}=0$. Let $j \in\{1, \ldots, k\}$, and let $I=\left[\tau_{j-1}, \tau_{j}\right]$. Let $\bar{\sigma}_{\ell_{1}}, \ldots, \bar{\sigma}_{\ell_{\sqrt{n}}}$ be the first $\sqrt{n}$ elements of $\bar{\sigma}$ that fall into $I$. Let $s=\max \left\{\tau_{j-1}, \sigma_{\ell_{\sqrt{n}}}\right\}$. Then the set $\left\{\bar{\sigma}_{\ell_{1}}, \ldots, \bar{\sigma}_{\ell_{\sqrt{n}}}\right\}$ is $\left(\left(\tau_{j}-\tau_{j-1}\right) n^{-1 / 4}\right)$-dense for the interval $\left[s, \tau_{j}\right]$ with a probability of at least $1-\exp \left(-\Theta\left(n^{1 / 4}\right)\right)$.

Proof. The claim follows from the first claim by setting $a=\tau_{j-1}, b=\tau_{j}$, and $y_{i}=\max \left\{a, \sigma_{\ell_{i}}\right\}$ for all $i \in\{1, \ldots, \sqrt{n}\}$. We have added the element $\tau_{0}=0$ so that the interval $\left[0, \tau_{1}\right]$ does not require special attention. Each $\bar{\sigma}_{\ell_{i}}$ is uniformly distributed in $\left[y_{i}, b\right]=\left[\max \left\{a, \sigma_{\ell_{i}}\right\}, \tau_{j}\right]$. Finally, $y_{i}=\max \left\{a, \sigma_{\ell_{i}}\right\} \in[a, s]=$ $\left[\tau_{j-1}, \max \left\{\tau_{j-1}, \sigma_{\ell_{\sqrt{n}}}\right\}\right]$ since $\sigma$ is a sorted sequence.

Let us return to our original aim: We wish to bound the length of an increasing run for which the values of the run all lie between the values of two consecutive left-to-right maxima. The idea is to apply Claim 2 twice. Each time, with high probability, if we consider additional $\sqrt{n}$ elements of the run, their values must be dense for a smaller and smaller interval. After having applied the claim twice, the interval is so small that, again with high probability, the interval can only contain a small number of elements - which proves that the total number of elements in the run cannot be large. In the following, we detail this argument.

Let $a=\bar{\sigma}_{i}$ be a left-to-right maximum of $\bar{\sigma}$, and let $b=\bar{\sigma}_{k}>a$ be the next left-to-right maximum after $a$, that is, $k>i$ is chosen minimally such that $\bar{\sigma}_{k}>a$. If less than $\sqrt{n}$ elements assume a value in $[a, b]$, then these elements contribute at most $\sqrt{n}$ to the length of an increasing run (recall that all elements of an increasing run must lie inside the interval $[a, b]$ if $a$ and $b$ are consecutive left-to-right maxima). Otherwise, we apply Claim 2 for $j=k$ : The first $\sqrt{n}$ 
elements of $\bar{\sigma}$ that are inserted into $[a, b]$ lie $\left((b-a) n^{-1 / 4}\right)$-densely in $[s, b]$ with a probability of at least $1-\exp \left(-\Theta\left(n^{1 / 4}\right)\right)$. If this not the case (despite the high probability), we call the situation a failure, which will be dealt with later.

Among the first $\sqrt{n}$ elements in $[a, b]$, let $c_{1}<\cdots<c_{m}$ be the elements in $[s, b]$ in increasing order. Let $c_{0}=s$ and $c_{m+1}=b$. Then, after inserting these $m$ elements into $[s, b]$, any increasing run whose elements assume values in $[s, b]$ can only be continued with elements of a subinterval $J=\left[c_{i-1}, c_{i}\right]$. The length $c_{i}-c_{i-1}$ of $J$ is at most $(b-a) n^{-1 / 4} \leq 2 n^{-1 / 4}$ since the first $\sqrt{n}$ elements are $\left((b-a) n^{-1 / 4}\right)$-dense in $[s, b]$. We apply the same argument once more: If less than $\sqrt{n}$ elements fall into $J$, then they contribute at most $\sqrt{n}$ to the length of an increasing run. Otherwise, we can apply Claim 2 again: The probability that after inserting $\sqrt{n}$ elements into $J$ there is a subinterval of $J$ of length at least $\left(c_{i}-c_{i-1}\right) n^{-1 / 4}$ that does not contain an element is at most $\exp \left(-\Theta\left(n^{1 / 4}\right)\right)$. If this nevertheless happens, we again call this a failure and deal with it later on.

We can now conclude that the values of all elements of an increasing run that we have not yet dealt with must lie in an interval of size at most $\left(c_{i}-c_{i-1}\right) n^{-1 / 4} \leq$ $2 n^{-1 / 2}$. The expected number of elements that fall into such an interval is at most $2 \sqrt{n}$. By the Chernoff bound, the probability that such an interval contains more than $4 \sqrt{n}$ elements is at most $\exp (-\Omega(\sqrt{n}))$. Again, we call it a failure if this nevertheless happens.

To finish the proof, we first estimate the length of the longest increasing run given that we have no failures. Second, we show that failures happen only with a negligible probability and contribute only little to the expected value of inc $(\bar{\sigma})$.

If we have no failure, then the expected length of any increasing run is at most $\mathbb{E}(\operatorname{ltrm}(\bar{\sigma}))$ plus $\sqrt{n}$ for the first $\sqrt{n}$ elements that fall between two consecutive left-to-right maxima plus $\sqrt{n}$ for the first $\sqrt{n}$ elements that fall into one interval of length at most $2 n^{-1 / 4}$ plus $4 \cdot \sqrt{n}$, which is the maximum number of elements in an interval of length $2 / \sqrt{n}$. Altogether, we have $\operatorname{inc}(\bar{\sigma}) \in O(\sqrt{n})$ in this case.

Failures can happen due to the following three events: First, there is an interval of length at most $2 n^{-1 / 4}$ between two left-to-right maxima that does not contain one of the first $\sqrt{n}$ elements that fall between these left-to-right maxima. Second, there is an interval of length at most $2 / \sqrt{n}$ that does not contain any of the first $\sqrt{n}$ elements after the second phase. Third, any interval of length $2 / \sqrt{n}$ contains more than $4 \sqrt{n}$ elements.

Overall, there are at most $O(n)$ pairs of consecutive left-to-right maxima between which a failure can happen. Furthermore, there are at most $O(n)$ pairs of consecutive elements $a$ and $b$ between which a failure can happen. Finally, there are at most $O(n)$ intervals of length at most $2 / \sqrt{n}$ where a failure can happen. (These are very rough estimates.) By taking a union bound, the overall probability of failure is thus at most $O\left(n \cdot \exp \left(-\Omega\left(n^{1 / 4}\right)\right)\right)$. If we have failure, then we bound $\operatorname{inc}(\bar{\sigma})$ by the trivial upper bound of $n$. This contributes only $O\left(n^{2} \cdot \exp \left(-\Omega\left(n^{1 / 4}\right)\right)\right) \subseteq o(1)$ to the expected value, which completes the proof of Lemma 8. 
Lemma 9. For every sequence $\sigma$, all $d$, and every covering $U_{1}, \ldots, U_{k}$ of the set $\{1, \ldots, n\}$ (which means $\bigcup_{i=1}^{k} U_{i}=\{1, \ldots, n\}$ ), we have

$$
\begin{aligned}
\operatorname{height}(\sigma) & \leq \sum_{i=1}^{k} \operatorname{height}\left(\sigma_{U_{i}}\right), \\
\mathbb{E}\left(\text { height }_{d}(\sigma)\right) & \leq \sum_{i=1}^{k} \mathbb{E}\left(\text { height }_{d}\left(\sigma_{U_{i}}\right)\right) .
\end{aligned}
$$

Proof. Let $U_{1}, \ldots, U_{k}$ cover $\{1, \ldots, n\}$. For a fixed $i$, let $a$ and $b$ with $a<b$ be two elements of $\sigma_{U_{i}}$ that do not lie on the same root-to-leaf path in $T\left(\sigma_{U_{i}}\right)$. Then there exists a $c$ prior to $a$ and $b$ in $\sigma_{U_{i}}$ with $a<c<b$, which implies that $a$ and $b$ do not lie on the same root-to-leaf path in the tree $T(\sigma)$ either. Now consider a root-to-leaf path $p$ of $T(\sigma)$ that has a length of height $(\sigma)$. Let $p_{U_{i}}$ be $p$ restricted to elements of $\sigma_{U_{i}}$ and let $\ell_{U_{i}}$ its length. Then $\sum_{i=1}^{k} \operatorname{height}\left(\sigma_{U_{i}}\right) \geq$ $\sum_{i=1}^{k} \ell_{U_{i}} \geq \operatorname{height}(\sigma)$, because the $U_{i}$ cover $\{1, \ldots, n\}$.

The second inequality follows directly from the first since taking expectation is a monotone operation.

Lemma 10. For every sequence $\sigma$ and for $d=n / \log ^{2} n$, we have

$$
\mathbb{E}\left(\text { height }_{d}(\sigma)\right) \in O(\log n) .
$$

Proof. Recall that a position $i$ is called eligible for an interval if $\bar{\sigma}_{i}$ could be any value in the interval, and it is called regular if it actually lies in the interval. All positions are eligible for $[1, d]$.

Let $\bar{\sigma}$ be a perturbed sequence and let $R$ be the set of regular positions with respect to $[1, d]$. A sufficient condition for $i$ being regular is $\nu_{i} \in[1, d-1]$. Let $F=\left\{i \in\{1, \ldots, n\} \mid \nu_{i} \leq 1\right\}$ and $B=\left\{i \in\{1, \ldots, n\} \mid \nu_{i} \geq d-1\right\}$ denote the sets of positions $i$ that are possibly not regular because $\nu_{i}$ is either too small or too large.

The three sets $R, F$, and $B$ are usually not disjoint, but they cover $\{1, \ldots, n\}$, which allows us to apply Lemma 9 in the following way: If we can individually bound the expected values of height ${ }_{d}\left(\sigma_{R}\right)$, height ${ }_{d}\left(\sigma_{F}\right)$ and height ${ }_{d}\left(\sigma_{B}\right)$ by $O(\log n)$, we are done.

Let us start with $\mathbb{E}\left(\right.$ height $\left.\left(\sigma_{R}\right)\right)$. Given that a position $i$ is regular, the element $\bar{\sigma}_{i}$ is uniformly distributed in $[1, d]$ and, thus, the order of the elements of $\bar{\sigma}_{R}$ is random with all permutations being equally likely. This implies that $\mathbb{E}\left(\right.$ height $\left.\left(\bar{\sigma}_{R}\right)\right) \in O(\log |R|) \subseteq O(\log n)$.

It remains to deal with $\bar{\sigma}_{F}$ and $\bar{\sigma}_{B}$. The distributions of height $\left(\bar{\sigma}_{F}\right)$ and height $\left(\bar{\sigma}_{B}\right)$ are clearly identical, so it suffices to analyze height $\left(\bar{\sigma}_{F}\right)$. For this, take a different look at how $\bar{\sigma}_{F}$ is generated: We can think of this as first flipping a coin for every $i \in\{1, \ldots, n\}$ to determine $i \in F$ with the coin being extremely biased so that $\mathbb{P}(i \in F)=1 / d=\left(\log ^{2} n\right) / n$ holds. After we have chosen $F$, we draw $\nu_{i}$ for each $i \in F$ uniformly at random from the interval [0,1].

By the Chernoff bound, the probability that $\bar{\sigma}_{F}$ contains more than $2 \log ^{2} n$ elements is less than $n^{-(\log n) / 3}$. If $\bar{\sigma}_{F}$ indeed contains more elements, we bound height $\left(\bar{\sigma}_{F}\right)$ by $n$. This contributes only $n^{-(\log n) / 3} \cdot n \in o(1)$ to the expected value of height $\left(\bar{\sigma}_{F}\right)$. 
We can apply Lemma 8 to $\sigma_{F}$, where $n^{\prime}=|F| \leq 2 \log ^{2} n$, and obtain $\mathbb{E}\left(\right.$ height $\left._{1}\left(\sigma_{F}\right)\right) \in O\left(\sqrt{n^{\prime}}\right) \subseteq O(\log n)$.

Lemma 11. For every sequence $\sigma$ and all $d \geq 1 / n$ we have

$$
\mathbb{E}\left(\text { height }_{d}(\sigma)\right) \in O(\sqrt{n / d}+\log n) .
$$

Proof. If $d \in \Omega\left(n / \log ^{2} n\right)$, then $\mathbb{E}\left(\right.$ height $\left._{d}(\sigma)\right) \in O(\log n)$ by Lemma 10 .

To prove the theorem for smaller values of $d$, we divide the sequence into subsequences. Let $N$ be the largest real root of the equation $N^{2} / \log ^{2} N=n d$. Then $\log N \in \Theta(\log (n d))$, and thus $N=c \cdot \sqrt{n d} \cdot \log (n d)$ for some $c \in \Theta(1)$. Let $n_{j}$ be the number of elements of $\sigma$ with $\sigma_{i} \in[(j-1) \cdot N / n, j \cdot N / n]$. Choose $k_{j} \in \mathbb{N}$ such that $\left(k_{j}-1\right) \cdot N<n_{j} \leq k_{j} N$. We divide the $n_{j}$ elements of the interval $[(j-1) \cdot N / n, j \cdot N / n]$ into $k_{j}$ subsequences $\sigma^{j, 1}, \ldots, \sigma^{j, k_{j}}$ such that no subsequence contains more than $N$ elements. Since

$$
\sum_{j=1}^{n / N} k_{j} \leq \sum_{j=1}^{n / N} \frac{n_{j}+N}{N} \leq 2 n / N
$$

we obtain at most $2 n / N$ such subsequences. Each subsequence spans at most an interval of length $N / n$ and contains at most $N$ elements. Thus, by Lemma 10, we have $\mathbb{E}\left(\right.$ height $\left._{d}\left(\sigma^{j, \ell}\right)\right) \in O(\log (N))$. Finally, Lemma 9 yields

$$
\mathbb{E}\left(\text { height }_{d}(\sigma)\right) \leq \sum_{j=1}^{n / N} \sum_{\ell=1}^{k_{j}} \mathbb{E}\left(\text { height }_{d}\left(\sigma^{j, \ell}\right)\right) \in O\left(\frac{n \log N}{N}\right)=O(\sqrt{n / d}) .
$$

\section{Smoothed Number of Quicksort Comparisons}

In this section, we apply our results on binary search trees and left-to-right maxima to the performance of the quicksort algorithm. The following theorem summarizes the findings.

Theorem 3. For $d \geq 1 / n$ we have

$$
\max _{\sigma \in[0,1]^{n}} \mathbb{E}\left(\mathrm{qs}_{d}(\sigma)\right) \in \Theta\left(\frac{n}{d+1} \sqrt{n / d}+n \log n\right) .
$$

In other words, if $d \in O(1)$, the number of comparisons is at most $O(n \sqrt{n / d})$, while if $d \in \Omega(1)$, it is at most $O\left(\frac{n}{d} \sqrt{n / d}\right)$. This means that $d$ has a stronger influence for $d \in \Omega(1)$.

\subsection{Upper Bound on the Smoothed Number of Quicksort Comparisons}

To prove the upper bound, we first need a lemma similar to Lemma 9 that allows us to estimate the number of comparisons of subsequences. 
Lemma 12. For every sequence $\sigma$, all $d$, and every covering $U_{1}, \ldots, U_{k}$ of the set $\{1, \ldots, n\}$, we have

$$
\begin{aligned}
\mathrm{qs}(\sigma) & \leq \sum_{i=1}^{k} \mathrm{qs}\left(\sigma_{U_{i}}\right)+Q, \\
\mathbb{E}\left(\mathrm{qs}_{d}(\sigma)\right)=\mathbb{E}(\mathrm{qs}(\bar{\sigma})) & \leq \sum_{i=1}^{k} \mathbb{E}\left(\mathrm{qs}\left(\bar{\sigma}_{U_{i}}\right)\right)+\mathbb{E}(\bar{Q}),
\end{aligned}
$$

where $Q$ is the number of comparisons of elements of $\sigma_{U_{i}}$ with elements of $\sigma_{\{1, \ldots, n\} \backslash U_{i}}$ for any $i$ and the random variable $\bar{Q}$ is defined analogously for $\bar{\sigma}$.

The proof goes along the same lines as the proof of Lemma 9 and is omitted.

Lemma 13. For every sequence $\sigma$ and all $d \geq 1 / n$, we have

$$
\mathbb{E}\left(\mathrm{qs}_{d}(\sigma)\right) \in O\left(\frac{n}{d+1} \sqrt{n / d}+n \log n\right) .
$$

Proof. First observe that quicksort will make at most $O(n \sqrt{n / d}+n \log n)$ comparisons, which follows directly from Lemma 11 and the fact that $\mathbb{E}(\mathrm{qs}(\bar{\sigma})) \leq$ $n \cdot \mathbb{E}($ height $(\bar{\sigma}))$ : Every level of recursion of quicksort contributes at most $n-1$ comparisons, and we have height $(\bar{\sigma})$ levels of recursion. Thus, the claim of the theorem is correct for $d \in O(1)$.

Let us now consider the case $d \in \omega(1)$. Furthermore, assume that $d \in$ $O\left(\sqrt[3]{n / \log ^{2} n}\right)$. This is no restriction since we obtain the average-case bound of $O(n \log n)$ already for $d \in \Theta\left(\sqrt[3]{n / \log ^{2} n}\right)$, thus also for larger $d$.

Similar to the proof of Lemma 10 , we divide the sequence $\bar{\sigma}$ into three parts. The set $R=\left\{i \in\{1, \ldots, n\} \mid \bar{\sigma}_{i} \in[1, d]\right\}$ of regular elements for the interval $[1, d]$ is defined as before. The set $F$ is defined slightly differently, namely as $F=\left\{i \in\{1, \ldots, n\} \mid \nu_{i} \leq 3\right\}$. This means that $F$ contains all $i$ for which $\nu_{i}$ is too small, plus some extra elements. Similarly $B=\left\{i \in\{1, \ldots, n\} \mid \nu_{i} \geq d-3\right\}$.

As in Lemma 10, the regular elements are easy to handle since they are uniformly distributed in $[1, d]$ and, thus, $\mathbb{E}\left(\mathrm{qs}_{d}\left(\sigma_{R}\right)\right) \in O(n \log n)$.

We have $\mathbb{E}\left(\right.$ height $\left._{d}\left(\sigma_{F}\right)\right)=\mathbb{E}\left(\right.$ height $\left._{d}\left(\sigma_{B}\right)\right) \in O(\sqrt{n / d})$, which follows from the same scaling argument that we used in Lemma 10 (see Lemma 1: The probability that $\sigma_{F}$ contains more than $6 n / d$ elements is at most $(e / 4)^{3 n / d} \in$ $O\left((e / 4)^{\sqrt{n}}\right)$ due to the Chernoff bound and $d \in O\left(\sqrt[3]{n / \log ^{2} n}\right)$. The same holds for $\sigma_{B}$. If either contains more elements, we bound the height by $n$, which contributes at most $o(1)$ to the expectation. Otherwise, we have sequences with $O(n / d)$ elements that are perturbed with a perturbation parameter of 3 . We obtain

$$
\mathbb{E}\left(\mathrm{qs}\left(\bar{\sigma}_{F}\right)\right)=\mathbb{E}\left(\mathrm{qs}\left(\bar{\sigma}_{B}\right)\right) \in O\left(\mathbb{E}\left(\text { height }_{3}\left(\bar{\sigma}_{F}\right)\right) \cdot n / d\right) \subseteq O\left(\frac{n}{d} \sqrt{n / d}\right) .
$$

By Lemma 12, what remains to be estimated is the number of comparisons of elements $\bar{\sigma}_{i}$ and $\bar{\sigma}_{j}$ where $i$ and $j$ are in two different sets of $R, F$, and $B$.

Due to the symmetry between $\bar{\sigma}_{F}$ and $\bar{\sigma}_{B}$, it suffices to restrict ourselves to estimating the number of comparisons of elements in $\bar{\sigma}_{F}$ with elements in $\bar{\sigma}_{R}$ and $\bar{\sigma}_{B}$. This boils down to counting the number of comparisons of elements $\bar{\sigma}_{i}$ with $\nu_{i} \leq 3$ to elements $\bar{\sigma}_{j}$ with $\bar{\sigma}_{j} \geq 1$. 
The number of comparisons between elements $\bar{\sigma}_{i}$ and $\bar{\sigma}_{j}$ with $i \in F$ and $j \in F \cap R$ can be bounded by the total number of comparisons between elements in $F$, but this number is $\mathbb{E}\left(\mathrm{qs}\left(\bar{\sigma}_{F}\right)\right) \in O\left(\frac{n}{d} \sqrt{n / d}\right)$. Similarly, since $\mathbb{E}\left(\mathrm{qs}\left(\bar{\sigma}_{R}\right)\right) \in$ $O(n \log n)$, the expected number of comparisons between positions $i \in F \cap R$ and $j \in R$ is at most $O(n \log n)$.

Thus, we can concentrate on $i \in F$ with $\nu_{i} \leq 1$ and $j \in R$ with $\bar{\sigma}_{j} \geq 3$, which includes all $i \in F \backslash R$ and $j \in R \backslash F$.

We distinguish two cases: First, we estimate the expected number of such comparisons with $\bar{\sigma}_{i}$ being the pivot element. Second, we consider the case that $\bar{\sigma}_{j}$ is the pivot element.

The two elements $\bar{\sigma}_{i} \leq \sigma_{i}+1 \leq 2$ and $\bar{\sigma}_{j} \geq 3$ will be compared with $\bar{\sigma}_{i}$ being the pivot only if $i<j$ and $\bar{\sigma}$ contains no element $\bar{\sigma}_{k} \in\left[\bar{\sigma}_{i}, \bar{\sigma}_{j}\right]$ for $k<i$. In particular, $\bar{\sigma}$ must not contain an element $\bar{\sigma}_{k} \in[2,3]$ with $k<i$.

Since $d \in \omega(1)$, every element is eligible for the interval $[2,3]$. Furthermore, for every $i \in\{1, \ldots, n\}$, we have $\mathbb{P}\left(\nu_{i} \leq 1\right)=\mathbb{P}\left(\bar{\sigma}_{k} \in[2,3]\right)=1 / d$ and these two events are disjoint. (If $\sigma_{i}=1$, then this is not true since it might be $\nu_{i}=1$. However, the probability of this is 0 .) Thus, the probability that $\bar{\sigma}$ contains more than $O(\log n)$ elements with $\nu_{i} \leq 1$ prior to the first element $\bar{\sigma}_{k} \in[2,3]$ is $O(1 / n)$. If this happens nevertheless, we bound the number of comparisons by the trivial upper bound of $n^{2}$, which contributes only $O\left(n^{2} \cdot 1 / n\right)=O(n)$ to the expected value.

Otherwise, at most $O(\log n)$ elements $\bar{\sigma}_{i}$ with $\nu_{i} \leq 1$ are compared to elements $\bar{\sigma}_{j}$ with $\bar{\sigma}_{j} \geq 3$ with $\bar{\sigma}_{i}$ being the pivot, which contributes $O(n \log n)$ comparisons.

Now we consider the second case: How many comparisons of elements $\bar{\sigma}_{j} \geq 3$ with elements $\bar{\sigma}_{i} \leq \sigma_{i}+1$ with $\bar{\sigma}_{j}$ being the pivot element do we have to expect? The element $\bar{\sigma}_{j}$ is compared to $\bar{\sigma}_{i}$ only if $j<i$ and there is no $k<j$ with $\bar{\sigma}_{k} \in\left[\bar{\sigma}_{i}, \bar{\sigma}_{j}\right]$. Thus, it is necessary that $\bar{\sigma}_{j}$ is the minimal among all elements $\bar{\sigma}_{k} \geq 3$ with $k \leq j$.

If we restrict ourselves to $\bar{\sigma}_{k} \in[3, d]$, then this corresponds just to the average number of left-to-right minima, which is $O(\log n)$. (The average number of leftto-right minima is equal to the average number of left-to-right maxima.) Thus, the expected number of elements $\bar{\sigma}_{j} \in[3, d]$ that, when being the pivot element, are compared to any element $\bar{\sigma}_{i} \leq \sigma_{i}+1$, is $O(\log n)$. This contributes at most $O(n \log n)$ to the expected number of comparisons.

Elements $\bar{\sigma}_{k} \geq d$ remain to be considered. Since $d \in \omega(1)$, there are at most $O(\log n)$ such elements prior to the first element of the interval $[3, d]$ with high probability. Furthermore, there are at most $O(\log n)$ elements of $\bar{\sigma}_{F}$ prior to the first element of $[1, d]$ with high probability. Thus, the contribution to the number of comparisons is only $O\left(\log ^{2} n\right)$. 


\subsection{Lower Bound on the Smoothed Number of Quicksort Comparisons}

The upper bound proved in the previous section is tight. The standard sorted sequence provides a worst case, but in the following lemma we use a sequence that is slightly easier to handle technically.

Lemma 14. For the sequence $\sigma=\left(1 / n, 2 / n, 3 / n, \ldots, \frac{n}{2} / n, 1,1, \ldots, 1\right)$ and all $d \geq 1 / n$, we have

$$
\mathbb{E}\left(\mathrm{qs}_{d}(\sigma)\right) \in \Omega\left(\frac{n}{d+1} \sqrt{n / d}+n \log n\right) .
$$

Proof. In the perturbed sequence $\bar{\sigma}$ the first $n / 2$ elements contain an expected number of $\Omega(\sqrt{n / d})$ left-to-right maxima according to Lemma 4 . Every left-toright maximum $\bar{\sigma}_{i}$ of $\bar{\sigma}$ has to be compared to all the elements that come later and are greater than $\bar{\sigma}_{i}$.

If $d \in o(1)$, all $n / 2$ elements of the second half of $\bar{\sigma}$ are greater than any left-to-right maximum of the first half of $\bar{\sigma}$. Thus, the expected number of comparisons is at least $\Omega(n \sqrt{n / d})=\Omega\left(\frac{n}{d+1} \sqrt{n / d}+n \log n\right)$.

If $d \in \Omega(1)$, then the probability that an element $\bar{\sigma}_{i}$ of the second half of $\bar{\sigma}$ is greater than all left-to-right maxima of the first half of $\bar{\sigma}$ is

$$
\mathbb{P}\left(\forall j \leq n / 2: 1+\nu_{i} \geq \bar{\sigma}_{j}\right) \geq \mathbb{P}\left(1+\nu_{i} \geq 1 / 2+d\right)=\frac{1}{2 d} .
$$

Thus, the expected number of elements that are greater than all left-to-right maxima of the first half is $\Omega(n / d)$. Multiplying this with the expected number of left-to-right maxima of the first half yields that at least an expected number of $\Omega\left(\frac{n}{d} \sqrt{n / d}\right) \subseteq \Omega\left(\frac{n}{d+1} \sqrt{n / d}\right)$ comparisons are needed. Since quicksort always needs at least $\Omega(n \log n)$ comparisons, we get the claim.

\section{Conclusion}

We have analyzed the smoothed height of binary search trees and the smoothed number of comparisons made by the quicksort algorithm under additive noise. The smoothed height of binary search trees and also the smoothed number of left-to-right maxima are $\Theta(\sqrt{n / d}+\log n)$; the smoothed number of quicksort comparisons is $\Theta\left(\frac{n}{d+1} \sqrt{n / d}+n \log n\right)$.

While we obtain the average-case height of $\Theta(\log n)$ for binary search trees only for $d \in \Omega\left(n / \log ^{2} n\right)$ - which is large compared to the interval size [0,1] from which the numbers are drawn -, for the quicksort algorithm $d \in \Omega\left(\sqrt[3]{n / \log ^{2} n}\right)$ suffices so that the expected number of comparisons equals the average-case number of $\Theta(n \log n)$. On the other hand, the recursion depth of quicksort, which is equal to the tree height, can be as large as $\Omega(\sqrt{n / d})$. Thus, although the average number of comparisons is already reached at $d \in \Omega\left(\sqrt[3]{n / \log ^{2} n}\right)$, the recursion depth remains asymptotically larger than its average value for $d \in$ $o\left(n /(\log n)^{2}\right)$. 
A natural question arising from our results is, what happens when the noise is drawn according to distributions other than the uniform distribution? In a more general additive noise model, the adversary can not only specify the sequence $\sigma$, but also a density function $f$ according to which the noise is drawn. We conjecture that if $\max _{x \in \mathbb{R}} f(x)=\phi$, then the expected tree height and the expected number of left-to-right maxima are $\Theta(\sqrt{n \phi}+\log n)$ while the expected number of quicksort comparisons is $\Theta\left(\frac{\phi n}{\phi+1} \sqrt{n \phi}+n \log n\right)$. These bounds would be in compliance with our bounds for uniformly distributed noise, where $\phi=1 / d$.

In our study of the quicksort algorithm we used the first element as the pivot element. This choice simplifies the analysis but is unrealistic since one would normally either use the middle element as the pivot element or use the median of the first, last, and middle element. Nevertheless, we conjecture that for both of these more realistic pivot strategies the same bounds as for the simple pivot strategy will result.

\section{References}

1. Cyril Banderier, René Beier, and Kurt Mehlhorn. Smoothed analysis of three combinatorial problems. In Branislav Rovan and Peter Vojtás, editors, Proc. of the 28th Int. Symp. on Mathematical Foundations of Computer Science (MFCS), volume 2747 of Lecture Notes in Computer Science, pages 198-207. Springer, 2003.

2. Michael Drmota. An analytic approach to the height of binary search trees II. Journal of the ACM, 50(3):333-374, 2003.

3. Michael Drmota. Profile and height of random binary search trees. Journal of the Iranian Statistical Society, 3(2):117-138, 2004.

4. James Allen Fill and Svante Janson. Quicksort asymptotics. Journal of Algorithms, 44(1):4-28, 2002.

5. Donald E. Knuth. Sorting and Searching, volume 3 of The Art of Computer Programming. Addison-Wesley, 2nd edition, 1998.

6. Bodo Manthey and Rüdiger Reischuk. Smoothed analysis of binary search trees. Theoretical Computer Science, 378(3):292-315, 2007.

7. Rajeev Motwani and Prabhakar Raghavan. Randomized Algorithms. Cambridge University Press, 1995.

8. Bruce Reed. The height of a random binary search tree. Journal of the ACM, 50(3):306-332, 2003.

9. John Michael Robson. Constant bounds on the moments of the height of binary search trees. Theoretical Computer Science, 276(1-2):435-444, 2002.

10. Robert Sedgewick. The analysis of quicksort programs. Acta Informatica, 7(4):327355, 1977.

11. Daniel A. Spielman and Shang-Hua Teng. Smoothed analysis of algorithms: Why the simplex algorithm usually takes polynomial time. Journal of the ACM, 51(3):385-463, 2004.

12. Daniel A. Spielman and Shang-Hua Teng. Smoothed analysis of algorithms and heuristics: Progress and open questions. In Luis M. Pardo, Allan Pinkus, Endre Süli, and Michael J. Todd, editors, Foundations of Computational Mathematics, Santander 2005, pages 274-342. Cambridge University Press, 2006. 